Revista de Psicología Vol. 30 (1), 2012 (ISSN 0254-9247)

\title{
Inteligencia cultural, valores y motivación para el aprendizaje en estudiantes militares argentinos
}

\author{
Pablo Domingo Depaula ${ }^{1}$ \\ Colegio Militar de la Nación, Argentina \\ Susana Celeste Azzollini ${ }^{2}$ \\ Conicet / Universidad de Buenos Aires, Argentina
}

El estudio tuvo como objetivo analizar si existen relaciones entre los niveles de inteligencia cultural desplegados por estudiantes militares argentinos, su motivación para el aprendizaje académico y los valores humanos vinculados al área laboral. Participaron 400 cadetes aspirantes a oficiales del Ejército Argentino (377 hombres y 23 mujeres) provenientes de diversas provincias argentinas. Se realizaron análisis de correlación bivariados a través del coeficiente $r$ de Pearson, cuyos resultados indican que los estudiantes presentan valores vinculados al cuidado de las tradiciones nacionales, al tiempo que el valor "estimulación" se asocia con la resolución creativa y original de experiencias nuevas o situaciones inciertas, equilibrando cierta auto-restricción que limitaría la apertura hacia la diversidad cultural.

Palabras clave: inteligencia cultural, motivación para el aprendizaje, valores humanos, estudiantes militares.

\section{Cultural intelligence, values and motivation to learn in Argentinean cadets}

The aim of this study was to analyze if relations among cultural intelligence levels, motivation to academic learning and human values on the working area in a sample of 400 argentine military students, cadets becoming officials on Argentine Army (377 men and 23 women), originating of diverse argentine provinces. Bivaried analyses of correlation through coefficient $r$ of Pearson were made. Results show that students present tie values to the care of the national traditions, and at the same time the value "stimulation" is associated to the creative resolution of new experiences on uncertain situations, balancing their autorestriction that would limit the opening towards cultural diversity.

Key words: Cultural intelligence, motivation to learn, human values, military students. 



\section{Aproximación teórica al estudio de la inteligencia cultural y los valores humanos en ámbitos militares}

El fenómeno de la globalización y su consecuente influencia sobre la cotidianeidad de las relaciones individuales y grupales, permite observar la diversidad de culturas que se enlazan al acontecer social e institucional de la época, trascendiendo los límites del conocimiento y las normas vigentes en cada nación. Bajo este panorama, la complejidad que caracteriza al contexto en el que se manifiestan las prácticas organizacionales en torno a un constante patrón de transformación local e internacional (Grosso, Mansilla \& Margarone, 2005), el auge de las comunicaciones ciberespaciales, el incremento de las relaciones establecidas entre diversos países del mundo en lo que respecta al ámbito político, económico, comunitario, científico, militar, etc., así como también las catástrofes climáticas, ambientales y bélicas, los actos de violencia, la discriminación ideológica, racial y religiosa, constituyen factores condicionantes del complejo y dinámico entramado cultural que configura la participación activa de cada continente en el marco de una red de intercambios multiculturales (Peterson, 2004).

Tales aspectos son especialmente importantes para los voluntarios que se encuentran desplegados operacionalmente en diversas regiones del mundo, ya que la toma de decisiones en situaciones de gran

1 Licenciado en Psicología y Magíster en Psicología Organizacional de la Universidad Abierta Interamericana. Doctorando en Psicología de la Universidad de Buenos Aires. Becario de Investigación del Ministerio de Defensa (Proyecto PIDDEF No 33). Docente / Investigador de la Secretaría de Investigación del Colegio Militar de la Nación. Contacto: Boquerón 973, Lomas de Zamora (1832), Provincia de Buenos Aires, Argentina; lic_pablodepaula@yahoo.com.ar

2 Doctora en Psicología de la UBA. Investigadora del Instituto de Investigaciones de la Facultad de Psicología. Investigadora Adjunta del Consejo Nacional de Investigaciones de Investigaciones Científicas y Técnicas (CONICET). Directora de proyectos UBACyT No P046 y PIDDEF No 33/10. Contacto: Fortunato Devoto 533, 6to piso, Ciudad Autónoma de Buenos Aires, Argentina; susana1060@yahoo.com.ar 
incertidumbre desencadena altos niveles de estrés y un uso adecuado de recursos para la resolución de problemas (Paparone \& Reed, 2008). Así, la participación en misiones de mantenimiento de la paz, ajustándose a los lineamientos y reglamentaciones de la Organización de las Naciones Unidas (ONU) con el fin de restaurar el bienestar de la población civil mediante la asistencia humanitaria, difiere del accionar convencional de los ejércitos tradicionales ( $\mathrm{Ng}$, Ramaya, Teo \& Wong, 2005), por lo que los procesos de aprendizaje y acción resultan de gran relevancia en la formación de los líderes militares actuales.

Bajo estos focos de acción internacional, la mayoría de las academias militares considera la importancia de ciertas cualidades involucradas en la formación y entrenamiento de sus estudiantes, las cuales exceden el área relativa a los rasgos de la personalidad por ejemplo, inclinándose hacia el desarrollo de habilidades multiculturales y de competencias que permitan el desempeño eficaz y la integración sociocultural y comunitaria en diversas regiones del mundo.

De hecho, según estudios realizados por Soeters y Bos-Bakx (como se cita en $\mathrm{Ng}$ et al., 2005) en dieciocho academias militares, a través de la utilización de las cuatro dimensiones culturales que integran la teoría de Hofstede (1980) (distancia al poder, evitación de la incertidumbre, individualismo-colectivismo, masculinidad-feminidad), se concluyó que en aquellas academias donde los factores culturales habían sido informados, la eficacia de las misiones resultó afectada de modo positivo. Por otra parte, Elron, Haley, Ben-Ari \& Shamir (2003) llevaron a cabo un estudio cualitativo recopilando datos de sesenta y dos entrevistas semi-estructuradas, con el objetivo de investigar el grado de cooperación y coordinación ejercida en determinadas operaciones de paz y evaluar la manera en que las conductas interculturalmente efectivas fueron fijadas dentro de las normas institucionales y mecanismos organizacionales. Como conclusión de tal estudio, las conductas observadas fueron categorizadas en tres factores: integración de diferencias, entrecruzamiento de diferencias y tolerancia a las diferencias.

A partir de estos resultados, se han suscitado discusiones respecto del entrenamiento cultural eficaz y acerca del modo en que las 
competencias culturales influyen sobre los niveles de efectividad de las misiones (Ng et al., 2005).

Considerando que un aspecto central de cualquier contexto cultural son los valores compartidos, Schwartz (1994) ha realizado una esquematización de las culturas de acuerdo a sus orientaciones en valores, proponiendo una teoría universal acerca de su contenido y su significado. Este autor los define como metas deseables, transituacionales, que varían en importancia y que sirven como principios en la vida de una persona o de otra entidad social. Estos motivan las acciones, funcionan como criterios que hacen posible juzgar y justificar acciones, adquiriéndose tanto por medio de la socialización a partir de un grupo dominante como mediante experiencias personales de aprendizaje.

Schwartz (1994) considera que, para poder adaptarse a la realidad sociocultural, los grupos y las personas transforman las necesidades propias de la existencia humana expresándolas en el lenguaje de los valores específicos mediante los que pueden comunicarse. Dicha transformación ocurre en respuesta a tres requisitos universales: las necesidades de los individuos en su condición de seres biológicos, la coordinación de las acciones sociales, la supervivencia y el correcto funcionamiento de los grupos. Asimismo, los valores individuales y culturales se hallan relacionados, dado que los últimos manifiestan ideas abstractas, compartidas socialmente, acerca de lo deseable de una sociedad o de un grupo. Bajo esta dinámica, las instituciones expresan tales valores mediante sus metas u objetivos, y sus líderes se ajustan a estos a través de un conjunto de creencias acerca del modo en que deberían ser y actuar (Thomas \& Inkson, 2003).

Específicamente, el modelo propuesto por Schwartz et al. (2001) establece diez tipos de valores, agrupados a través de cuatro metas generales, a saber: Estimulación, Autodirección y Hedonismo (agrupados en la meta general apertura al cambio); Tradición, Conformidad y Seguridad (agrupados en la meta general conservación); Universalismo y Benevolencia (agrupados en la meta general trascendencia) y; Poder y Logro (agrupados en la meta general autopromoción) (Castro Solano, 2005). 
En suma, tales modelos de comprensión transcultural, que legitiman la formación y entrenamiento multicultural tanto de militares como de civiles, justifican la transformación y reorganización de las fuerzas armadas modernas hacia la adopción de nuevos roles en un vasto espectro de operaciones ( $\mathrm{Ng}$ et al., 2005). Sosteniendo tal premisa organizacional, el ejercicio del liderazgo militar implica la identificación de una conciencia cultural o competencia cultural, necesaria para el óptimo despliegue de operaciones militares en contextos culturales diversos (Graen \& Hui, 1999; Gurstein, 1999; Shamir \& Ben-Ari, 1999; Yukl, como se cita en Ng et al., 2005).

En virtud de la importancia asignada al desarrollo de estas competencias multiculturales, se ha venido otorgando un interés creciente el concepto de inteligencia cultural. Las primeras definiciones teóricas en torno a la misma establecían que se trataba de ciertas capacidades personales para adaptarse de manera efectiva a nuevos contextos culturales (Earley \& Ang, 2003), involucrando cuatro factores dimensionales extrapolados de los múltiples locus de inteligencia propuestos por Sternberg (1986). Dichos factores son: la inteligencia cultural cognitiva, relativa a conocimientos que permiten la comprensión de las normas de interacción social de cada cultura singular; la inteligencia cultural metacognitiva, referida a aquellas estrategias que permiten adquirir cada conocimiento, construir y mantener las relaciones interculturales; la inteligencia cultural motivacional, representante del anhelo y la confianza por impulsar y reiterar relaciones con individuos pertenecientes a diferentes culturas y; por último, la inteligencia cultural conductual, que se manifiesta a través de la implementación de conductas apropiadas a la diversidad cultural a fin de establecer relaciones más efectivas. Estos cuatro factores han sido aplicados en gran medida en operaciones globales diversas y en métodos de entrenamiento cultural.

Posteriormente, Ang et al. (2007) redefinieron tal constructo teórico, conceptualizándolo como la capacidad individual para entenderse eficazmente con personas que difieren en sus orígenes y conocimientos culturales. Dicho concepto ha sido introducido en la literatura de la última década como un continuo cuantitativo de diferencias 
individuales cuya definición ha carecido de especificidad de constructo, en virtud de haber alcanzado cierta vaguedad en torno a similitudes conceptuales con otros términos como el de competencias interculturales, de ser considerado como un modo de pensamiento global o bien como una extensión descriptiva de un nuevo dominio del constructo de inteligencia social (Thomas et al., 2008).

Una de las más recientes y elaboradas definiciones postula que la inteligencia cultural constituye un sistema de interacción de conocimientos y habilidades conectadas mediante metacognición cultural, cuya habilidad conciente y deliberada de monitoreo y regulación de procesos cognitivos y estados afectivos relacionados con objetivos precisos, permite a las personas adaptarse, seleccionar y configurar los aspectos culturales de su ambiente (Thomas et al., 2008), siendo este último factor una función de enlace entre los conocimientos y habilidades culturales que configuran tal sistema metacognitivo.

\section{La motivación para el aprendizaje en estudiantes militares}

Las variables motivacionales se encuentran entre las más abordadas en relación con el rendimiento de los estudiantes en el sistema educativo formal, y en lo que atañe a la evaluación y optimización de los procesos de aprendizaje subyacentes a tal rendimiento. Alonso Tapia (1997), para evaluar motivacionalmente el desempeño de los estudiantes, propone la indagación de cuatro metas de aprendizaje académico: las relacionadas con el valor de logro (competencia), las relacionadas con el valor intrínseco (aprendizaje y dominio de conocimiento), las relacionadas con el valor instrumental (ligadas a móviles externos), y las relacionadas con los valores negativos (rechazo).

Desde una perspectiva teórica similar, Deci y Ryan (1985) distinguen varios tipos de motivación relacionados con las diferentes metas que guían los comportamientos personales, diferenciando entre motivación intrínseca y varios tipos de motivación extrínseca. La motivación intrínseca proporciona una mayor calidad de las experiencias 
de aprendizaje, ya que los estudiantes están orientados por móviles en relación con el dominio y la adquisición del conocimiento y no por metas externas a ellos; provocando, a su vez, un sentido de dominio y competencia (Castro Solano, 2005).

Con relación a los aspectos motivacionales vinculados a los procesos de aprendizaje, Bandura (1999a) argumenta que gran parte de la motivación humana se genera cognitivamente, en virtud de que las personas se motivan a sí mismas y dirigen sus acciones de forma anticipada, elaborando creencias sobre lo que pueden hacer, imaginando los resultados probables o acciones futuras en función del establecimiento de objetivos para sí mismas y planificando cursos de acción orientados al logro eficaz de tales metas, a través de la movilización de cierto nivel de voluntad y esfuerzo. Los motivadores cognitivos que operan para el logro de la eficacia corresponden a atribuciones causales, expectativas de resultado y metas cognitivas.

Según este autor, en lo que respecta a la teoría social cognitiva,"las personas deben desarrollar destrezas a fin de regular los determinantes motivacionales, afectivos y sociales de su funcionamiento intelectual así como los aspectos cognitivos. Esto implica desempeñar influencia sobre todos los aspectos del proceso de aprendizaje" (Bandura, 1999a, p. 35).

Zimmerman (como se cita en Bandura, 1999a) ha desarrollado estudios acerca de un modelo ampliado de auto-regulación académica, aunque Bandura afirma que las destrezas de auto-regulación no son tan influyentes si los estudiantes no logran persistir frente a las dificultades, estresores o atracciones competitivas. De hecho, la creencia firme en las propias destrezas auto-reguladoras facilita la perseverancia académica favoreciendo el rendimiento satisfactorio de los estudiantes.

Pozo Municio (2003) señala que, si bien los móviles para intentar un aprendizaje pueden ser extrínsecos o intrínsecos, con frecuencia se produce una combinación entre ambos; por lo que resulta relevante, desde el punto de vista de la instrucción, promover inicialmente la motivación de manera más eficaz y duradera, procurando que el aprendiz sea menos dependiente de factores externos, lo cual se relaciona con el deseo de aprender (Alonso Tapia, 1992). Al respecto, Pozo Municio 
(2003) considera que "de hecho, la polaridad extrínseco-intrínseco debe entenderse como un continuo, de modo que generar un deseo por aprender es de algún modo hacer que el aprendiz vaya interiorizando (o atribuyéndose a sí mismo) motivos que inicialmente percibiera afuera” (pp. 176-177).

Nótese que la idea de este autor acerca del deseo de aprender como resultado de la interiorización o auto-atribución de los motivos externos, se halla en consonancia con los aportes de Bandura (1999b) acerca de la auto-eficacia como factor de activación de procesos motivacionales, además de cognitivos, afectivos y de selección, de forma tal que mediante feedback, las percepciones de auto-eficacia reforzarían el componente desiderativo del aprendizaje.

Por su parte, Oettingen (1999), en línea con los aportes dimensionales de Hofstede (1980) en relación al concepto de cultura, argumenta que las diferencias culturales pueden provocar diferentes valoraciones de la auto-eficacia, pudiendo expresarse en las prácticas sociales del sistema familiar y educativo. Concluye lo siguiente:

La investigación en diferentes culturas de las creencias de auto-eficacia clarifica que las creencias de eficacia se originan bajo diferentes prácticas sociales e institucionales. Apunta hacia el poder de las instituciones de la sociedad, las cuales, de formas culturalmente determinadas, modifican la prevalencia, forma y evaluación de diferentes fuentes de información sobre la auto-eficacia (p. 152).

Este autor agrega que, en relación a la motivación académica, las valoraciones que efectúan las personas respecto a su eficacia se hallan fuertemente influidas por las comparaciones sociales, siendo esto atribuible especialmente a los contextos educativos donde las ejecuciones académicas se hallan sujetas, en mayor medida, al modelado y a la evaluación comparativa.

De hecho, Castro Solano y Fernández Liporace (2005) analizaron la influencia de componentes motivacionales y cognitivos sobre el rendimiento en estudiantes militares argentinos, y encontraron que aquellos que presentaban un menor interés en el contenido de las asignaturas 
vinculados a aspectos netamente académicos más que instructivo-militares, se hallaban orientados por una motivación más extrínseca y, por lo tanto, obtenían bajos rendimientos. Tal resultado ha permitido a los autores reforzar la idea vinculada a la importancia que revisten los factores motivacionales en la planificación de las actividades de aprendizaje en este tipo de estudiantes.

El principal objetivo de esta investigación es indagar los valores humanos y las metas motivacionales de aprendizaje que manifiestan los estudiantes militares argentinos durante su formación y su relación con sus niveles de inteligencia cultural.

En referencia a las hipótesis del estudio, se plantea que: a) el interés y la reflexión sobre la diversidad cultural (representados por la dimensión metacognitiva o reflexiva-motivacional de la inteligencia cultural) se asocian a metas de aprendizaje académico dirigidas hacia el logro de la autonomía personal (es decir, hacia metas de aprendizaje/dominio de los conocimientos); en tanto que b) las metas aversivas externas, materializadas en la repulsión por el objeto de conocimiento, obstaculizan la motivación para relacionarse con personas provenientes de otras culturas (es decir la inteligencia cultural reflexiva-motivacional); y c) la inteligencia cultural, expresada en mayor medida a través de la reflexión y las motivaciones por conocer y adaptarse a la cultura (factor reflexivomotivacional), se asocia al valor humano tradición (perteneciente a la meta valórica conservación), lo que implica que los estudiantes militares priorizan durante su formación como subtenientes del Ejército el cuidado de las tradiciones de su patria, sus símbolos y la defensa nacional.

\section{Método}

Se diseñó una investigación correlacional delimitando las siguientes variables principales: inteligencia cultural, motivación para el aprendizaje y valores humanos que portan los estudiantes militares. 


\section{Participantes}

La muestra del estudio estuvo integrada por 400 cadetes aspirantes a oficiales del Ejército Argentino, quienes durante los meses de agosto y septiembre del año $2008^{3}$ cursaban la Licenciatura en Conducción y Gestión Operativa en el Colegio Militar de la Nación. Se seleccionó una muestra no probabilística por cuotas según el año en curso, considerándose 100 cadetes de cada uno de los cuatro años de la carrera. El $94.3 \%(n=377)$ de los cadetes eran de sexo masculino y el $5.8 \%(n=23)$ de sexo femenino. La edad promedio era de 21.71 años $(D E=2.37$ años).

Por otro lado, el $98.2 \%$ de los estudiantes $(n=393)$ eran nacidos en Argentina en las siguientes regiones geográficas: $45.3 \%$ de los cadetes $(n=181)$ eran nacidos en la región Pampa, $14.5 \%(n=58)$ en el Noroeste Argentino, $14.3 \%(n=57)$ en la Mesopotamia, 8.5\% $(n=34)$ en las Sierras Pampeanas, 7.8\% $(n=31)$ en la región de Cuyo, $6.3 \%$ $(n=25)$ eran oriundos de la Patagonia y $1.8 \%(n=7)$ de la región de Chaco. El $1.8 \%$ de los cadetes restantes $(n=7)$ habían nacido en el exterior del país. La totalidad de los estudiantes $(N=400)$ residían al momento de la recolección de datos en la región Pampa, dado el régimen de internado semanal que requiere la cursada académica e instructiva en el instituto de formación militar.

Con respecto al arma o especialidad de la que forman parte los estudiantes (por elección voluntaria o por orden de mérito - cuando existe una gran cantidad de cadetes que eligen determinadas armas o especialidades- $)$ el 25\% $(n=100)$ pertenecía al Cuerpo Comando, es decir aquellos cursantes del primer año de la carrera que aún no han elegido arma o especialidad, dado que tal determinación se lleva a cabo una vez que alcanzan el segundo año; el $17.5 \%(n=70)$ de los cadetes pertenecía al arma de Infantería, el $14 \%(n=56)$ a Caballería,

3 El tiempo transcurrido desde la realización de la investigación hasta su publicación se explica porque el presente estudio corresponde a algunos de los objetivos específicos abordados en la Tesis de Maestría en Psicología Organizacional realizada por Depaula (2010a), la cual debía contener información inédita. 
el $12.5 \%(n=50)$ integraba la especialidad de Ingenieros, el $11.8 \%$ $(n=47)$ pertenecía al arma de Artillería, el $9.5 \%(n=38)$ integraba la especialidad de Comunicaciones, el 6\% $(n=24)$ la de Intendencia y el $3.8 \%(n=15)$ la de Arsenales.

\section{Instrumentos}

Encuesta Sociodemográfica (Depaula, 2010a)

Es un instrumento diseñado ad hoc para el presente estudio, cuyo propósito es indagar los siguientes datos de los participantes: sexo, siendo los indicadores 'masculino' y 'femenino'; edad, contada en años; lugar de nacimiento, que releva datos sociodemográficos correspondientes a las siguientes regiones geográficas que integran la República Argentina: Pampa, Estepa, Noroeste, Chaco, Sierras Pampeanas, Mesopotamia, Cuyo y Patagonia, y Exterior del País; año de cursada correspondiente a la Licenciatura en Conducción y Gestión Operativa (carrera universitaria de grado que se articula a la instrucción militar básica recibida por los aspirantes a oficiales del Ejército Argentino); arma o especialidad, que integran los cadetes en la organización militar a la que pertenecen, siendo los indicadores correspondientes a las armas: Infantería, Artillería, Ingenieros, Comunicaciones y Caballería; y los indicadores de las especialidades: Arsenales e Intendencia; Profesional (que contempla los servicios de Aviación del Ejército, Sanidad, Veterinaria, Justicia, Computación de Datos y Educación Física); Cuerpo Comando, siendo este último indicador correspondiente a los estudiantes del primer año que no integran ningún arma o especialidad hasta alcanzar el segundo año de la cursada.

\section{Cultural Intelligence Scale (CQS) (Ang et al., 2007)}

Esta escala está compuesta por 20 ítems que evalúan las cuatro dimensiones que componen a la inteligencia cultural (Earley \& Ang, 2003), a saber, a) dimensión metacognitiva, que refleja los procesos mentales que los individuos utilizan para adquirir y comprender el 
conocimiento cultural, incluyendo el control de conocimientos y pensamientos relativos a la cultura, e integra capacidades que incluyen la planificación, monitoreo y revisión de modelos mentales relativos a normas culturales pertenecientes a países o grupos de personas; b) dimensión cognitiva, que evalúa el conocimiento de normas, prácticas y convenciones adquiridas en diferentes culturas, producto de la educación y las experiencias personales, incluyendo conocimientos relativos a los sistemas económicos, legales y sociales de diferentes culturas y subculturas, y conocimientos acerca del marco de valores culturales que distingue a cada cultura en particular; c) dimensión motivacional, que refleja la capacidad de dirigir la atención y la energía hacia el aprendizaje y funcionamiento en situaciones caracterizadas por diferencias culturales como un agente de control de afectos, cogniciones y conductas que facilitan el logro de objetivos; y, c) dimensión conductual, que permite evaluar la capacidad para exhibir apropiadamente acciones verbales y no-verbales interactuando con personas de diferentes culturas, a través de valores culturales propios de contextos específicos, incluyendo un vasto repertorio de conductas.

Los reactivos se responden mediante una escala con formato Likert con 7 opciones de respuesta, pero en el actual estudio el formato contempló 5 opciones cuyo escalamiento presentaba las categorías entre Muy en desacuerdo/En parte en desacuerdo/Ni de acuerdo ni en desacuerdo/En parte de acuerdo/Muy de acuerdo, a fin de reducir los tiempos de administración. El cuestionario fue diseńado por los autores originales para ser auto-administrado en forma individual o colectiva.

En lo que respecta a los criterios de validez y confiabilidad de la prueba, Depaula (2010a) realizó nuevos análisis factoriales exploratorios de componentes principales con rotación Promax del instrumento de Ang et al. (2007) traducido al castellano. Obtuvo una solución factorial definitiva que consta de tres factores que explican el $50.9 \%$ de la varianza (Test de Esfericidad de Bartlett $=2284,104 ; p<.01$ ) (Índice Kaiser Meyer Olkin = 0.84). El proceso fue llevado a cabo de forma iterativa, eliminándose tres de los ítems provenientes de la escala original, dado que inicialmente se presentaban niveles de fiabilidad 
significativamente bajos en uno de los factores (integrado por dos ítems originales) resultantes de la estructura factorial preliminar $(\alpha=.28)$, al tiempo que en la estructura factorial definitiva se eliminó un ítem que presentaba cargas factoriales similares en más de un factor.

Los resultados mostraron que la última versión del instrumento presenta propiedades psicométricas aceptables. En efecto, ha arrojado niveles meritorios de fiabilidad $(\alpha=.81)$. De un posterior análisis de la fiabilidad realizado a través del modelo de dos mitades (split), también han resultado niveles aceptables de fiabilidad (valor $\alpha$ para la parte $1=.78$; valor $\alpha$ para la parte $2=.71$ ).

En virtud de los análisis estadísticos mencionados, la escala utilizada en el presente estudio se halla integrada por las siguientes dimensiones: inteligencia cultural reflexiva-motivacional ( $\alpha=.77)$; inteligencia cultural cognitiva $(\alpha=.79)$; inteligencia cultural conductual-lingüística $(\alpha=.79)$.

\section{Escala de Metas de Aprendizaje, Población Militar (EMA-M)}

(Castro Solano \& Casullo, 2002)

La escala EMA-M consiste en una operacionalización del modelo de motivación para el aprendizaje postulado por Alonso Tapia (como se cita en Castro Solano, 2005), el cual considera que las metas del aprendizaje pueden ser internas y/o externas. En la misma línea, los aportes de Bandura (1999a) vinculados a la auto-eficacia y la motivación académica resultan centrales para la evaluación y el análisis de esta variable. Este instrumento puede ser utilizado únicamente en población de estudiantes militares, ya que las frases reflejan el contenido relacionado con la educación académica aplicada en tal ámbito. El cuestionario fue diseñado para ser auto-administrado, pudiendo administrarse en forma individual o colectiva. Los reactivos se responden mediante una escala con formato Likert con 5 opciones de respuesta, cuyo escalamiento presenta las categorías Muy en desacuerdo/En parte en desacuerdo/Ni de acuerdo ni en desacuerdo/En parte de acuerdo/Muy de acuerdo. 
Las dimensiones evaluadas a través de la prueba se definen conceptualmente de la siguiente forma: a) metas en relación con el aprendizaje y el dominio de los conocimientos, es decir que se aprende por el deseo de aprender en sí mismo, porque tiene un valor en sí mismo, por satisfacción personal o por el deseo de dominar algún aspecto del conocimiento, sin buscar obtener algo a cambio; b) metas aversivas externas, significando que las consecuencias producto del estudio son las cuestiones importantes a considerar y no el aprendizaje en sí mismo, manifestándose aversión o repulsión por el objeto de conocimiento; y, c) metas de lucimiento, que indican que se aprende para ser aceptado, para quedar bien con los demás, ya sea con profesores o instructores militares, es decir ser valorado por sus superiores.

Los autores de la prueba verificaron la estructura factorial obtenida inicialmente a través de un análisis factorial confirmatorio encontrando, en población militar más que en civil, un mejor ajuste de la estructura factorial al modelo teórico elegido. Por otro lado, los autores obtuvieron fiabilidades satisfactorias para cada uno de los tres factores, a saber: metas en relación con el aprendizaje y dominio $(\alpha=.70)$; metas aversivas externas $(\alpha=.73)$; $y$, metas en relación con el lucimiento $(\alpha=.65)$.

Cuestionario de Valores Contextualizados (VAL) (Castro Solano, 2005;

Casullo \& Castro Solano, 2004)

Es una escala auto-administrable dispuesta de 30 ítems que evalúan los valores en dominios específicos para población adulta. Está basado en la topología de diez valores de Schwartz et al. (2001) mencionada anteriormente, con el propósito de evaluar la orientación de los valores de las personas en la recreación y vida social, en las relaciones familiares y en el trabajo. Estas tres áreas de orientación y evaluación valórica constituyen formalmente las tres partes en que se presenta el instrumento, cada una de las cuales consta de diez ítems sobre los que la persona tiene que autoevaluarse en relación con el comportamiento descrito por cada frase (Castro Solano, 2005). En el presente estudio se administró solamente la Parte 3 del cuestionario, es decir la relativa 
al trabajo, puesto que se decidió evaluar la contextualización valórica de los estudiantes militares en el área laboral en que se desempeñarán en su futuro profesional. Los ítems que integran dicha parte plantean situaciones que el sujeto debe valorar según una escala de 1 a 5 de acuerdo con sus preferencias, expresando el número 5 su máxima preferencia y el 1 la menor; el número 3, señala un punto intermedio de preferencia en la escala.

Los diez tipos motivacionales de valores que define conceptualmente la teoría constituyen las dimensiones del instrumento, agrupándose en cuatro metas generales que permiten interpretar los datos resultantes de la administración; a saber: a) apertura al cambio, representante de la valoración de la independencia en acciones, pensamientos, sentimientos y nuevas experiencias, agrupando los siguientes valores: Estimulación (importan la novedad y los desafíos), Autodirección (interés por poder pensar de manera independiente, tener libertad de acción y exploración, por ser creativo) y Hedonismo (importancia del placer y la gratificación personal, a fin de lograr disfrutar de la vida); b) conservación, la que implica autorrestricción, orden y seguridad, hallándose integrada por los siguientes valores: Tradición (interesa el respeto y el compromiso con las costumbres, con la cultura tradicional y con la religión), Conformidad (se valoran las normas sociales y se evitan los comportamientos que puedan herir o molestar a los demás, se respeta a los mayores) y Seguridad (interesan la seguridad, la armonía y la estabilidad sociales y personales); c) trascendencia o autotrascendencia, vinculada a la valoración del bienestar e interés por los demás, agrupando los siguientes valores: Universalismo (importan la tolerancia y la justicia social, la protección del medio ambiente) y Benevolencia (importa preservar e intensificar el bienestar de las personas, la honestidad y la ausencia de rencor); y, d) autopromoción, meta valórica que supone la valoración de los intereses personales, siendo integrada por los siguientes valores: Poder (interés por lograr poder social, autoridad, riqueza) y Logro (búsqueda del éxito personal poniendo en juego competencias que son socialmente aceptables). 
Por último, el instrumento fue adaptado y validado por sus autores. En efecto, el cálculo de la consistencia de los valores a través del coeficiente alfa de Cronbach para cada una de las dimensiones que agrupan a los tipos de valores y para cada una de las poblaciones (civiles, cadetes y oficiales), les permitió verificar que las dimensiones conservación y trascendencia resultaron las más consistentes para los tres tipos de poblaciones (rango entre .60 y .70 aproximadamente), al tiempo que las dimensiones autopromoción y apertura al cambio fueron las menos consistentes para los tres tipos de poblaciones (rango entre .50 y .60) sin arrojar discrepancias significativas.

\section{Procedimiento}

Los datos fueron analizados mediante el soporte técnico del paquete informático SPSS 11.0, llevando a cabo análisis de correlación lineal a través del Coeficiente $r$ de Pearson. Para analizar los niveles críticos de significación asociados a tal coeficiente estadístico, se ha aceptado un error $\alpha<.05$.

\section{Resultados}

\section{Correlaciones entre inteligencia cultural y motivación para el aprendizaje}

Los resultados arrojados por el análisis de correlación lineal evidencian que existe una correlación positiva y estadísticamente significativa entre la inteligencia cultural reflexiva-motivacional y las metas de dominio/aprendizaje $(r=.39 ; p=.000)$. Tales valores estadísticos indican que cuanto más altos son los niveles de reflexión e interés por adaptarse a la diversidad cultural, más se utilizan metas de aprendizaje orientadas hacia el óptimo desarrollo del potencial académico del estudiante, cuyos procesos desplegados durante la formación radican en componentes motivacionales intrínsecos que predispondrían al logro de cierta autonomía personal. 
Por otro lado, aunque con un coeficiente más difícil de interpretar, existiría una correlación negativa entre la dimensión reflexiva motivacional de la inteligencia cultural y las metas aversivas externas $(r=-.18$; $p=.000)$, indicando que cierta tendencia repulsiva hacia la obtención de un rendimiento académico meritorio por parte de los estudiantes podría obstaculizar el interés y la reflexión sobre la eventual posibilidad de establecer relaciones interculturales y viceversa.

\section{Tabla 1}

Relaciones entre la inteligencia cultural y la motivación para el aprendizaje de estudiantes militares

\begin{tabular}{|c|c|c|c|c|c|}
\hline & & $\begin{array}{l}\text { Inteligencia } \\
\text { cultural } \\
\text { reflexiva- } \\
\text { motivacional }\end{array}$ & $\begin{array}{l}\text { Inteligencia } \\
\text { cultural } \\
\text { cognitiva }\end{array}$ & $\begin{array}{l}\text { Inteligencia } \\
\text { cultural } \\
\text { conductual- } \\
\text { lingüística }\end{array}$ & $\begin{array}{c}\text { Inteligencia } \\
\text { cultural } \\
\text { (general) }\end{array}$ \\
\hline \multirow{2}{*}{$\begin{array}{l}\text { Metas de } \\
\text { aprendizaje/ } \\
\text { dominio }\end{array}$} & $r$ & $.39^{* *}$ & $.15^{* *}$ & -.05 & $.20^{* *}$ \\
\hline & Sig. (bilateral) & .000 & .002 & .336 & .000 \\
\hline \multirow{2}{*}{$\begin{array}{l}\text { Metas de } \\
\text { lucimiento }\end{array}$} & $r$ & -.07 & .09 & $.16^{* *}$ & $.11^{*}$ \\
\hline & Sig. (bilateral) & .187 & .065 & .001 & .034 \\
\hline \multirow{2}{*}{$\begin{array}{l}\text { Metas } \\
\text { aversivas } \\
\text { externas }\end{array}$} & $r$ & $-.18^{* *}$ & -.096 & .054 & -.09 \\
\hline & Sig. (bilateral) & .000 & .055 & .282 & .072 \\
\hline \multirow{2}{*}{$\begin{array}{l}\text { Motivación } \\
\text { para el } \\
\text { aprendizaje } \\
\text { (general) }\end{array}$} & $r$ & .03 & .070 & $.114^{*}$ & $.11^{*}$ \\
\hline & Sig. (bilateral) & .598 & .160 & .022 & .035 \\
\hline
\end{tabular}

Nota. $N=400 ;{ }^{*} p \leq .05$ (bilateral); ${ }^{* *} p \leq .01$ (bilateral).

\section{Correlaciones entre inteligencia cultural y valores humanos en el trabajo}

Con respecto a las relaciones entre la inteligencia cultural y los valores humanos en el trabajo, se ha evidenciado una correlación positiva y significativa entre la inteligencia cultural (general) y la meta general 
conservación $(r=.32 ; p=.000)$, lo cual indicaría que las habilidades de adaptación a contextos culturales diversos que portan los estudiantes se manifiestan en un marco de autorrestricción, orden y seguridad sobre sí mismos. En este sentido, la meta general conservación ha correlacionado también con la dimensión reflexiva-motivacional de la inteligencia cultural $(r=.34 ; p=.000)$, lo cual da cuenta de que la adaptación a la diversidad cultural deviene, en cierta forma, de la reflexión y la motivación de los estudiantes por mantener la protección de sí mismos y el reaseguro del ambiente frente al contexto divergente. Considerando particularmente los valores humanos que integran esta dimensión, se observa que el valor Tradición (es decir aquello relacionado con el interés y ocupación por la religión, las tradiciones y las costumbres regionales del país de origen de los estudiantes) correlaciona positivamente con la inteligencia cultural cognitiva $(r=.27 ; p=.000)$ y con la inteligencia cultural reflexiva motivacional $(r=.27 ; p=.000)$, lo cual indicaría que las relaciones interculturales desplegadas por los estudiantes priorizan en cierta forma el cuidado y la defensa de la soberanía nacional mediante el ejercicio del sentido patriótico que subyace a la profesión militar, mecanizando la reflexión, el interés y el conocimiento de las tradiciones nacionales, de la propia historia, el ejercicio de la memoria colectiva, el respeto y equilibrio del orden institucional, y las costumbres regionales de su país de origen.

El significativo predominio de la meta general conservación mencionado en el párrafo anterior (acentuado en la autorrestricción y el interés por la propia cultura), en general podría encontrar cierto equilibrio a través de la polaridad valórica opuesta, es decir la meta general apertura al cambio. No obstante, el valor Estimulación (integrante de esta última meta valórica) ha presentado correlaciones positivas poco significativas con la inteligencia cultural conductuallingüística $(r=.14 ; p<.01)$ y con la inteligencia cultural cognitiva $(r=.13 ; p<.01)$. Analizando el comportamiento de la polaridad valórica conservación-apertura al cambio, las apreciaciones estadísticas anteriores indicarían que, mientras los estudiantes transitan el devenir de un proceso de adaptación a la Institución Ejército, focalizarían sus 
intereses vocacionales en la internalización y protección de los símbolos e instituciones propias de la cultura nacional, de su país de origen, más que en la reflexión acerca de los rasgos particulares y desconocidos de otras culturas propias del contexto internacional.

Por otro lado, en lo que refiere a la meta general autopromoción, se observa una correlación positiva entre el valor Logro y la inteligencia cultural reflexiva-motivacional $(r=.39 ; p=.000)$, lo cual indicaría que el monitoreo de pensamientos, acciones, conocimientos y el interés de los estudiantes por operar sobre la diversidad cultural se relaciona con la búsqueda del éxito, constituyendo un genuino desafío personal y poniendo en juego competencias socialmente aceptables; al tiempo que el valor Poder, es decir, el hecho de buscar destacarse frente al resto de los estudiantes movilizado por motivaciones extrínsecas de estudio, se manifiesta en una escasa medida de significación, sobre todo a través de conductas espontáneas frente a la diversidad, poco mediadas por la reflexión y la motivación intrínseca por comprender y adaptarse a la cultura ajena $(r=.10 ; p<.05)$. No obstante, infiriendo acerca de la dinámica dada entre la polaridad valórica autopromoción-autotrascendencia, puede apreciarse que la inteligencia cultural reflexiva-motivacional correlaciona positivamente con el valor Benevolencia $(r=.31 ; p=.000)$, significando en los estudiantes un alto nivel de comprensión e interés por aceptar la diversidad cultural procurando llevar a cabo tareas de relevancia social para ayudar a quienes sufren o presentan problemas serios; al tiempo que la misma dimensión de la inteligencia cultural correlaciona positivamente, pero en menor medida, con el valor Universalismo $(r=.23$; $p=.000)$, implicando cierta tendencia hacia el despliegue de la reflexión y el interés sobre conocimientos y acciones culturales que permitan realizar tareas u operaciones relacionadas con la protección de la naturaleza y el mejoramiento de la calidad de vida de aquellos que pertenecen a una cultura diferente. 


\section{Tabla 2}

Relaciones entre la inteligencia cultural y los valores humanos en el trabajo de estudiantes militares

\begin{tabular}{|c|c|c|c|c|c|}
\hline & & $\begin{array}{l}\text { Inteligencia } \\
\text { cultural } \\
\text { reflexiva- } \\
\text { motivacional }\end{array}$ & $\begin{array}{c}\text { Inteligencia } \\
\text { cultural } \\
\text { cognitiva }\end{array}$ & $\begin{array}{l}\text { Inteligencia } \\
\text { cultural } \\
\text { conductual- } \\
\text { lingüística }\end{array}$ & $\begin{array}{c}\text { Inteligencia } \\
\text { cultural } \\
\text { (general) }\end{array}$ \\
\hline \multirow{2}{*}{ Universalismo } & $r$ & $.23^{* *}$ & $.19^{* *}$ & .08 & $.23^{* *}$ \\
\hline & Sig. (bilateral) & .000 & .000 & .09 & .000 \\
\hline \multirow{2}{*}{ Benevolencia } & $r$ & $.31^{* *}$ & $.16^{* *}$ & .05 & $.23^{* *}$ \\
\hline & Sig. (bilateral) & .000 & .001 & .37 & .000 \\
\hline \multirow{2}{*}{$\begin{array}{l}\text { Meta general } \\
\text { autotrascendencia }\end{array}$} & $r$ & $.14^{* *}$ & .10 & .01 & $.10^{*}$ \\
\hline & Sig. (bilateral) & .007 & .05 & .90 & .04 \\
\hline \multirow{2}{*}{ Tradición } & $r$ & $.27^{* *}$ & $.27^{* *}$ & .08 & $.28^{* *}$ \\
\hline & Sig. (bilateral) & .000 & .000 & .12 & .000 \\
\hline \multirow{2}{*}{ Conformidad } & $r$ & .07 & $.11^{*}$ & .04 & $.10^{*}$ \\
\hline & Sig. (bilateral) & .16 & .03 & .46 & .05 \\
\hline \multirow{2}{*}{ Seguridad } & $r$ & $.17^{* *}$ & .06 & -.03 & .08 \\
\hline & Sig. (bilateral) & .001 & .27 & .51 & .13 \\
\hline \multirow{2}{*}{$\begin{array}{l}\text { Meta general } \\
\text { conservación }\end{array}$} & $r$ & $.34^{* *}$ & $.28^{* *}$ & .09 & $.32^{* *}$ \\
\hline & Sig. (bilateral) & .000 & .000 & .07 & .000 \\
\hline \multirow{2}{*}{ Poder } & $r$ & .05 & $.13^{* *}$ & $.10^{*}$ & $.14^{* *}$ \\
\hline & Sig. (bilateral) & .32 & .01 & .04 & .01 \\
\hline \multirow{2}{*}{ Logro } & $r$ & $.39^{* *}$ & $.16^{* *}$ & -.02 & $.22^{* *}$ \\
\hline & Sig. (bilateral) & .000 & .002 & .69 & .000 \\
\hline \multirow{2}{*}{$\begin{array}{l}\text { Meta general } \\
\text { autopromoción }\end{array}$} & $r$ & .07 & .04 & $.12^{*}$ & $.11^{*}$ \\
\hline & Sig. (bilateral) & .17 & .49 & .01 & .03 \\
\hline \multirow{2}{*}{ Hedonismo } & $r$ & -.07 & .00 & .10 & .03 \\
\hline & Sig. (bilateral) & .18 & .98 & .05 & .60 \\
\hline \multirow{2}{*}{ Estimulación } & $r$ & .07 & $.13^{* *}$ & $.14^{* *}$ & $.17^{* *}$ \\
\hline & Sig. (bilateral) & .17 & .01 & .004 & .001 \\
\hline \multirow{2}{*}{ Autodirección } & $r$ & -.07 & -.04 & $.12^{*}$ & .02 \\
\hline & Sig. (bilateral) & .17 & .44 & .01 & .69 \\
\hline \multirow{2}{*}{$\begin{array}{l}\text { Meta general } \\
\text { apertura al cambio }\end{array}$} & $r$ & .07 & $.16^{* *}$ & $.14^{* *}$ & $.18^{* *}$ \\
\hline & Sig. (bilateral) & .17 & .002 & .004 & .000 \\
\hline
\end{tabular}

Nota. $N=400 ;{ }^{*} p \leq .05$ (bilateral); ${ }^{* *} p \leq .01$ (bilateral). 


\section{Discusión}

Los movimientos migratorios internos y externos (de los que participan estudiantes nacidos en Buenos Aires - provincia donde se localiza el instituto bajo estudio- y estudiantes oriundos del interior de la Argentina y de otros países limítrofes) y la integración a la organización de formación militar confluyen en un mutuo proceso de aculturación (Berry, 1974, 1999), lo cual requiere necesariamente el despliegue dinámico de la inteligencia cultural.

Particularmente, el lugar de relevancia que ocupa la motivación en la activación y el despliegue de la inteligencia cultural, resulta coincidente con los aportes de Kanfer y Heggestad (1997; Depaula, 2010a), quienes consideran que tales capacidades motivacionales operan proveyendo un agente de control de afectos, cogniciones y conductas que facilitan el logro de objetivos. Paralelamente, algunos autores destacan la importancia de la responsabilidad en lo que respecta a los procesos de aprendizaje. Rivière (1992) refiriéndose a la teoría social cognitiva y a la teoría de la auto-eficacia postulada por Bandura (1999a, 1999b), establece que tal aspecto individual participa activamente en los procesos de aprendizaje a través de mecanismos auto-reguladores de carácter motivacional, concediendo al pensamiento una importancia esencial en el control de la conducta. Bajo esta perspectiva de monitoreo autoreflexivo sobre el propio acto de aprender, la metacognición cultural (Thomas et al., 2008) adquiere jerarquía en relación a los procesos de aprendizaje que se desarrollan en el marco de un ambiente institucional multicultural; en tanto el despliegue de conductas imitativas frente a patrones culturales divergentes, se asociaría a metas de aprendizaje centradas en la búsqueda del logro académico mediante conductas de lucimiento motivadas extrínsecamente en relación a docentes y superiores.

En síntesis, la capacidad auto-reguladora y la capacidad de auto-reflexión, mecanizadas por la inteligencia cultural reflexivamotivacional frente a la diversidad, deberían operar progresivamente durante la formación-instrucción de los futuros líderes militares, pro- 
curando incrementar la valoración por los contextos culturales diversos. Bajo esta óptica, el pensamiento humano vinculado a la adquisición y despliegue de habilidades culturales con el auxilio de la inteligencia cultural alcanza una importancia capital siendo este concebido como un instrumento que incrementa la capacidad de adaptarse de forma eficaz al entorno, dado que implica la representación y manipulación simbólica de los acontecimientos y sus interrelaciones (Rivière, 1992), los cuales emergen cotidianamente en el devenir académico e instructivo militar.

Centrando el foco en los resultados arrojados por los análisis de correlación entre la inteligencia cultural, las metas de aprendizaje y los valores humanos, se puede observar el modo en que los estudiantes militares, quienes se hallan transitando un período de formación de carrera académica e instrucción militar que precede a la posibilidad de ser destinados a diversas guarniciones militares localizadas en las diferentes regiones geográficas de la Argentina, o bien su eventual participación voluntaria en misiones de paz internacionales representando como cascos azules a las Naciones Unidas, presentan en gran medida valores vinculados al cuidado de las tradiciones nacionales. Pero puntualmente, durante el mencionado período pre-laboral, se hallan en segundo plano los valores vinculados a la independencia en acciones, pensamientos, sentimientos, y al despliegue de conocimientos culturales, idioma y comportamientos que posibiliten la búsqueda de nuevas experiencias transculturales, no siendo debidamente impulsados — se infiere- hasta tanto el joven oficial experimente más cercanamente la posibilidad de participar de una misión de paz, por ejemplo, en una cultura diferente a la propia o bien sea destinado a trabajar en cuarteles ubicados en regiones geográficas diferentes a la de origen.

En cierta medida, la conservación o autorrestricción cultural que podría obstaculizar el contacto cultural (Berry, 1974, 1999) debiera trascender progresivamente los límites personales y la gran adherencia a las tradiciones nacionales, promoviendo cierta apertura al cambio en las relaciones socioculturales, en situaciones de incertidumbre que demandarían resoluciones originales, nuevas, creativas, sin depen- 
der necesariamente de un esquema o modelo de toma de decisiones estrictamente predeterminado. En esta línea, el fortalecimiento académico e instructivo militar del valor Estimulación (asociado al despliegue de conocimientos y conductas culturalmente adaptativas) podría fomentar la presencia y el ejercicio de un estilo de liderazgo transformacional (Bass \& Avolio, 1990), en el cual predomine su correspondiente sub-dimensión estimulación intelectual, facilitando la apertura a la creatividad e innovación frente a situaciones inciertas (Depaula, 2010b).

En este sentido, se puede plantear un potencial conflicto en la formación militar vinculado a dos roles claramente diferenciados: el tradicional de defensa del territorio nacional, para el cual se requiere la valoración de los propios usos y costumbres y la subordinación a órdenes impartidas por superiores, y otro actualizado de participación en misiones de paz y humanitarias en otros países, para el cual es necesaria la apertura a otras culturas, valoración del multiculturalismo y un pensamiento crítico en relación a la toma de decisiones y la capacidad de negociación.

Los resultados alcanzados respecto a la meta general autopromoción, representada por el valor Logro, han enfatizado el anhelo de los estudiantes hacia la búsqueda del éxito personal a través de competencias socialmente aceptables (Castro Solano, 2005), lo cual resulta esperable en los estudiantes en actual formación.

En suma, tales hallazgos destacan la relevancia que cobra el fenómeno de la globalización y la consecuente necesidad de que los futuros líderes militares porten motivaciones vocacionales genuinas, valores humanos y habilidades culturales que les permitan adaptarse a los entornos cambiantes de la época en las diversas regiones del mundo. Bajo esta premisa, resulta legítimo desarrollar nuevas investigaciones analizando empíricamente la posibilidad de brindar una educación que fomente las características necesarias para el desempeño de ambos roles señalados. 


\section{Referencias}

Alonso Tapia, J. (1992). (Ed.). Motivar en la adolescencia. Teoría, evaluación e intervención. Madrid: Servicio de Publicaciones de la Universidad Autónoma.

Alonso Tapia, J. (1997). Orientación educativa. Teoría, evaluación e intervención. Madrid: Síntesis.

Ang, S., Van Dyne, L., Koh, C., Ng, K. Y., Templer, K. J., Tay, C. et al. (2007). Cultural intelligence: Its measurements and effects on cultural judgments and decision making, cultural adaptation and task performance. Management and Organization Review, 3(3), 335-371.

Bandura, A. (1999a). Ejercicio de la eficacia personal y colectiva en sociedades cambiantes. En A. Bandura (Ed.), Auto-eficacia: cómo afrontamos los cambios de la sociedad actual (pp. 19-54). Bilbao: Desclée De Brouwer.

Bandura, A. (1999b). (Ed.), Auto-Eficacia: Cómo afrontamos los cambios de la Sociedad actual. Bilbao: Desclée de Brouwer.

Bass, B. M. \& Avolio, B. (1990). Manual de Multifactor Leadership Questionnaire. Palo Alto, CA: Consulting Psychologists Press.

Berry, J. W. (1974). Psychological aspect of cultural pluralism: Unity and identity reconsidered. Topics in Culture Learning, 2, 17-22.

Berry, J. W. (1999). Intercultural relations in plural societies. Canadian Psychology, 40(1), 12-21.

Castro Solano, A. (2005). Técnicas de evaluación psicológica en los ámbitos militares. Buenos Aires: Paidós.

Castro Solano, A. \& Casullo, M. M. (2002). Razonamiento, motivación, intereses profesionales y rendimiento académico en cadetes militares. Revista Iberoamericana de Evaluación y Diagnóstico Psicológico, 13(1), 83-101.

Castro Solano, A. \& Fernández Liporace, M. (2005). Predictores para la selección de cadetes en instituciones militares. Psykhe, 14(1), 17-30. 
Casullo, M. M. \& Castro Solano, A. (2004). Valores humanos y contextos en población civil y militar. Acción Psicológica (UNED), 3(1), 21-30.

Deci, E. \& Ryan, R. (1985). Intrinsic motivation and self-determination in human behavior. Nueva York: Plenum.

Depaula, P. (2010a). Integración de predictores globales del rendimiento en estudiantes de un instituto militar. Desarrollos y aplicaciones de inteligencia cultural para la defensa (Tesis de maestría inédita). Universidad Abierta Interamericana, Buenos Aires, Argentina.

Depaula, P. (N2010b). Inteligencia cultural y estilos de liderazgo en estudiantes de un instituto de formación militar. En Memorias del II Congreso Internacional de Investigación y Práctica Profesional en Psicología, XVII Jornadas de Investigación y Sexto Encuentro de Investigadores en Psicología del MERCOSUR (Tomo 4, pp. 86-88). Buenos Aires: Universidad de Buenos Aires.

Earley, P. C. \& Ang, S. (2003). Cultural intelligence: Individual interactions across cultures. Stanford, CA: University Press.

Elron, E., Haley, N., Ben-Ari, E. \& Shamir, B. (2003). Cooperation and coordination across cultures in the peacekeeping forces: Individual and organizational integrating mechanisms. En T. W. Britt \& A. B. Adler (Eds.), The psychology of the peacekeeper (pp. 261-282). Westpoint, CT: Praeger.

Graen, G. B. \& Hui, C. (1999). U.S. Army leadership in the twentyfirst century: Challenges and implications for training. En J. G. Hunt, G. E. Dodge \& L. Wong (Eds.), Out-of-the-box leadership: Transforming the twenty-first century army and other top performing organizations (pp. 15-40). Stamford, CT: Jai Press.

Grosso, F., Mansilla, J. \& Margarone, V. (2005). Claves para el desarrollo de la empresa. Buenos Aires: Dunken.

Gurstein, M. (1999). Leadership in the peacekeeping army of the future. En J. G. Hunt, G. E. Dodge \& L. Wong (Eds.), Out-ofthe-box leadership: Transforming the twenty-first century army and other top performing organizations (pp. 15-40). Stamford, CT: Jai Press. 
Hofstede, G. (1980). Culture's consequences: International differences in work-related values. Newbury Park, CA: Sage.

Kanfer, R. \& Heggestad, E. D. (1997). Motivational traits and skills: A person-centered approach to work motivation. Research in Organizational Behavior, 19, 1-56.

Ng, K. Y., Ramaya, R., Teo, T. M. S. \& Wong, S. F. (2005). Cultural intelligence: Its potential for military leadership development. Recuperado de http://www.imta.info/PastConferences/Papers. aspx? ShowAll=True

Oettingen, G. (1999). Perspectivas interculturales sobre auto-eficacia. En A. Bandura (Ed.), Auto-eficacia: cómo afrontamos los cambios de la sociedad actual (pp. 131-153). Bilbao: Desclée De Brouwer.

Paparone, C. R. \& G. Reed (2008). The reflective military practitioner: How military professionals think in action. Military Review, 2(88), 66-76.

Peterson, B. (2004). Cultural intelligence: A guide to working with people from other cultures. Boston: Intercultural Press.

Pozo Municio, J. I. (2003). Aprendices y maestros. La nueva cultura del aprendizaje. Madrid: Alianza.

Rivière, A. (1992). La teoría social del aprendizaje. Implicaciones educativas. En C. Coll, J. Palacios \& A. Marchesi (Eds.), Desarrollo psicológico y educación (Vol. 2, pp. 1-10). Madrid: Alianza.

Schwartz, S. (1994). Are there universal aspects in the structure and content of human values? Journal of Social Issues, 50, 19-45.

Schwartz, S., Melech, G., Lehman, A., Burgess, S., Harris, M. \& Owens, V. (2001). Extending the cross-cultural validity of the theory of basic human values with a different method of measurement. Journal of Cross Cultural Psychology, 32(5), 519-542.

Shamir, B. \& Ben-Ari, E. (1999). Leadership in an open army. En J. G. Hunt, G. E. Dodge \& L. Wong (Eds.), Out-of-the-box leadership: Transforming the twenty-first century army and other top performing organizations (pp. 15-40). Stamford, CT: Jai Press.

Sternberg, R. J. (1986). A framework for understanding conceptions of intelligence. En R. J. Sternberg \& D. K. Detterman (Eds.), 
What is intelligence? Contemporary viewpoints on its nature and definition (pp. 3-15). Norwood, NJ: Ablex.

Thomas, D. C. \& Inkson, K. (2003). Cultural intelligence. People skills for global business. San Francisco, CA: Berrett-Koehler.

Thomas, D. C., Elron, E., Stahl, G., Ekenlund, B. Z., Ravlin, E. C., Cerdin, J. et al. (2008). Cultural intelligence: Domain and assessment. International Journal of Cross Cultural Management, 2(8), 123-143.

Zimmerman, B. J. (1990). Self-regulating academic learning and achievement: The emergence of a social cognitive perspective. Educational Psychology Review, 2, 173-201.

Recibido: 28 de julio, 2011 Aceptado: 30 de octubre, 2011 